\title{
ANALYTICAL CIRCLE GROUP ACTIONS ON COMPACT COMPLEX MANIFOLDS ${ }^{1}$
}

\author{
BY SHAW MONG \\ Communicated by Glen E. Bredon, July 12, 1972
}

1. Introduction. Let $M$ be a compact complex manifold (of $m$ complex dimensions), and let $G$ be a compact Lie group acting analytically on $M$. Then the Dolbeault complexes

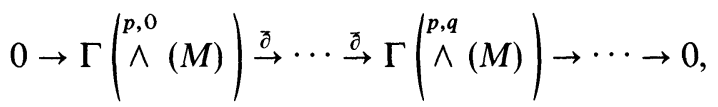

$p=0, \ldots, m$, are $G$-elliptic complexes (for the definitions and following notions see [1], [2], [3]) and their analytical indices $\chi\left(A^{p, *}, G\right)$ (or simply $\left.\chi^{p}\right)$ are elements in the group representation $\operatorname{ring} R(G)$. Following Hirzebruch [4], we have the $\chi_{y}\left(A^{p, *}, G\right)$ (or $\chi_{y}$ )-characteristic, $\sum_{p=0}^{m} \chi^{p}(-y)^{p}$ (here we take the alternating sum rather than the sum in [4]), which is an element in $R(G)[y]$.

Let $\mathscr{C}_{k}$ be the category of $(M, G)$ such that $M$ has $k$ fixed points under the analytical action of $G$, and let $\mathscr{C}=\bigcup_{k=0}^{\infty} \mathscr{C}_{k}$. In this note we study the category $\mathscr{C}_{k}, k=2,3$, for the case $G=S^{1}$. (Note: (i) $\mathscr{C}_{1}=\varnothing$ and (ii) $\chi_{y}=0$ for $\left(M, S^{1}\right) \in \mathscr{C}_{0}$.) Precisely the problem is: what are the necessary conditions for $\left(M, S^{1}\right) \in \mathscr{C}_{k}, k=2,3$, and if they do exist, what is their $\chi_{y}$ and the representations of $S^{1}$ on the tangent planes over the fixed point set? The main tools for this study are the $S^{1}$-index theory and Atiyah-Bott fixed point formula. Only the statement of the result is given here. The details of the proof will appear elsewhere.

\section{Main theorems.}

THEOREM 1. If $\left(M, S^{1}\right) \in \mathscr{C}$, then $\chi_{y} \in Z[y]$. Furthermore, if at a fixed point $A$, the representation of $S^{1}$ on the tangent plane $T_{A} M$ is given by $T_{A} M(t)=t^{a_{1}}+\ldots+t^{a_{m}}$, where $t \in R\left(S^{1}\right)=Z\left[t, t^{-1}\right]$, then

$$
\chi_{y}=\sum_{S^{1}(A)=A} \prod_{i=1}^{m}\left(\frac{1-y t^{a_{i}}}{1-t^{a_{i}}}\right) \text {. }
$$

THEOREM 2. If $\left(M, S^{1}\right) \in \mathscr{C}_{2}$, then either (i) $M=S^{2}$ or (ii) (complex) dim $M$ $=3$.

AMS (MOS) subject classifications (1970). Primary 58G10, 57D25.

Key words and phrases. $G$-index, Atiyah-Bott fixed point formula, $\chi_{y}$-characteristic, analytic actions, representation rings.

${ }_{1}^{1}$ This research was supported by NSF grant GU3171. 
Corollary. Let $\left(M, S^{1}\right) \in \mathscr{C}_{2}$.

(i) If $M=S^{2}$, then $(*)$ is given by

$$
1+y=\frac{1-y t^{a}}{1-t^{a}}+\frac{1-y t^{-a}}{1-t^{-a}}
$$

where $a$ is a nonzero integer.

(ii) If (complex) $\operatorname{dim} M=3$, then $(*)$ is given by

$$
\begin{aligned}
y+y^{2}= & \left(\frac{1-y t^{-a-b}}{1-t^{-a-b}}\right)\left(\frac{1-y t^{a}}{1-t^{a}}\right)\left(\frac{1-y t^{b}}{1-t^{b}}\right) \\
& +\left(\frac{1-y t^{a+b}}{1-t^{a+b}}\right)\left(\frac{1-y t^{-a}}{1-t^{-a}}\right)\left(\frac{1-y t^{-b}}{1-t^{-b}}\right)
\end{aligned}
$$

where $(a, b)$ is a pair of positive integers.

A simple example for case (i) is given by $S^{1}$ acting on $S^{2}$ as a rotation along the axis through north and south poles on $S^{2}$. It is an interesting problem arising from (ii) that if given any pair $(a, b)$ of positive integers, can we find an analytical $S^{1}$-action of case (ii) type?

TheOREM 3. If $\left(M, S^{1}\right) \in \mathscr{C}_{3}$, then $M$ must be a complex surface and (*) is given by

$$
\begin{aligned}
1+y+y^{2}= & \left(\frac{1-y t^{a}}{1-t^{a}}\right)\left(\frac{1-y t^{b}}{1-t^{b}}\right) \\
& +\left(\frac{1-y t^{a-b}}{1-t^{a-b}}\right)\left(\frac{1-y t^{-b}}{1-t^{-b}}\right)+\left(\frac{1-y t^{b-a}}{1-t^{b-a}}\right)\left(\frac{1-y t^{-a}}{1-t^{-a}}\right)
\end{aligned}
$$

where $a \neq b$ are any nonzero integers.

ExAMPLE. The linear action of $S^{1}$ on $C P(2)$ given by: $\left(z_{0}, z_{1}, z_{2}\right) \rightarrow$ $\left(z_{0}, t^{a} z_{1}, t^{b} z_{2}\right), a \neq b$, belongs to $\mathscr{C}_{3}$.

\section{REFERENCES}

1. M. F. Atiyah and R. Bott, The Lefschetz fixed point formula for elliptic complexes. I, II, Ann. of Math. (2) 86 (1967), 374-407; ibid. (2) 88 (1968), 451-491. MR 35 \# 3701; MR 38 \#731.

2. M. F. Atiyah and I. M. Singer, The index of elliptic operators. I, Ann. of Math. (2) 87 (1968), 484-530. MR 38 \# 5243.

3. M. F. Atiyah and G. B. Segal, The index of elliptic operators. II, Ann. of Math. (2) 87 (1968), 531-545. MR 38 \# 5244.

4. F. Hirzebruch, Topological methods in algebraic geometry, 3rd ed., Die Grundlehren der math. Wissenschaften, Band 131, Springer-Verlag, New York, 1966. MR 34 \# 2573.

Department of Mathematics, State University of New York at Albany, Albany, NEW YORK 12203

(Current address): Departement de Mathématiques, Université de Montréal, Montréal 101, Québec, Canada 